\title{
Improved In Vitro Fertilization Ability of Mouse Sperm Caused by the Addition of Licorice Extract to the Preincubation Medium
}

\author{
Naguyen H. Tung ${ }^{1}$, Yukihiro Shoyama ${ }^{1}$, Morimasa Wada ${ }^{2}$ and Hiromitsu Tanaka ${ }^{2, *}$ \\ ${ }^{I}$ Pharmacognosy, and ${ }^{2}$ Molecular Biology Laboratories, Faculty of Pharmaceutical Sciences, Nagasaki International \\ University, Huis Ten Bosch 2825-7 Sasebo, Nagasaki Japan 859-3298, Japan
}

\begin{abstract}
Artificial fertilization is used for the stable production of domestic animals, conservation of rare animals, and infertility treatment. However, the success rate of artificial fertilization is limited; thus, a more effective approach is desired. Cattle workers in Japan often add licorice grass to the herbage given to their animals before performing artificial insemination in an effort to increase the pregnancy rate. To examine the effects of licorice on fertilization success in mice, an aqueous extract of licorice was added to the culture medium used for insemination. The results were compared to those using glycyrrhizin, a major active constituent of licorice. The fertilization rate was improved by the aqueous licorice extract, but not specifically by glycyrrhizin. Thus, flavonoids in licorice other than glycyrrhizin may improve the success rate of artificial fertilization.
\end{abstract}

Keywords: Assisted reproductive technology (ART), embryo, herbal medicine, infertility, sperm.

\section{INTRODUCTION}

Licorice (genus Glycyrrhiza) root has medicinal properties and is used in at least $70 \%$ of traditional Chinese medicine formulae; it is used as a treatment agent for various diseases, including gastrospasm, stomach ache, sore throat, gastric ulcers, and duodenal ulcers [1]. Licorice is also widely used as a sweetener in the production of confectioneries and soy sauce. Nearly 500 compounds have been identified in licorice root; among them, glycyrrhizin and several flavonoids are major components [1]. The global demand for licorice is high.

In vitro fertilization (IVF) has been established in mice, and many mouse lines have been preserved as embryos or fertilized eggs. By performing IVF using cryopreserved or long-term-refrigerated sperm, mouse lines can be preserved more easily. Although previous studies have investigated whether licorice can increase pregnancy rates, no study has shown that it is effective [2].

An ejected sperm begins moving immediately, but it does not have inherent fertilization ability [3]. That is, each sperm must first complete maturation by undergoing capacitation and the acrosome reaction. Knowledge of the molecular mechanisms of sperm maturation in vivo is limited. Although natural pregnancy is possible, it is currently difficult to obtain a fertilized egg by IVF [4]. Hence, this process must be improved. Here, we report that the IVF rate in mice can

*Address correspondence to this author at the Molecular biology Laboratory, Faculty of Pharmaceutical Sciences, Nagasaki International University, Huis Ten Bosch 2825-7 Sasebo, Nagasaki 859-3298, Japan; Tel/Fax:+81-956-20-5651, E-mail: h-tanaka@niu.ac.jp be improved by cultivating sperm in the water-soluble fraction of a licorice extract before insemination.

\section{MATERIALS AND METHODS}

\section{Animals}

Ten-week-old female ICR mice were purchased from Kyudo IBL (Kumamoto, Japan). BALB/cA or C57BL/6 mice were purchased from Japan SLC (Shizuoka, Japan). All animal experiments conformed to the Guide for the Care and Use of Laboratory Animals of Nagasaki International University (Nagasaki, Japan) and were approved by the Institutional Committee of Laboratory Animal Experimentation (Nagasaki International University). The mice were kept under controlled temperature $\left(18-26^{\circ} \mathrm{C}\right)$ and lighting (12:12 h light-dark cycle) conditions throughout the experiments and were provided food and water ad libitum.

\section{Preparation of the Licorice Extract}

Licorice (Glycyrrhiza uralensis) roots cultivated in Genkai-cho, Saga Prefecture, Japan, were collected in August of 2011 and stored at the Faculty of Pharmaceutical Science, Nagasaki International University. An air-dried sample $(350 \mathrm{~g})$ was sliced and then extracted with hot distilled water $(500 \mathrm{~mL} \times 8 \mathrm{~h} \times 3$ times $)$ at $40^{\circ} \mathrm{C}$ under sonication. The combined extracts were filtered and concentrated under reduced pressure. The residue obtained was lyophilized to produce a crude aqueous licorice extract (deep yellow powder, $51.8 \mathrm{~g}$ ).

The crude aqueous extract was suspended in water then partitioned successively with ethylacetate and n-butanol. 
The ethylacetate and n-butanol fractions were evaporated under reduced pressure to obtain residues. Each fraction was then solubilized to a concentration of $3 \mathrm{mg} / \mathrm{mL}$ in dimethyl sulfoxide (DMSO).

\section{Sperm Collection}

Mice were sacrificed by cervical dislocation just before the start of the experiments. Mature caudal epididymal sperm $\left(\sim 8 \times 10^{6}\right)$ from each mouse were incubated in $200 \mu \mathrm{L}$ of human tubal fluid (HTF) medium without bovine serum albumin (BSA) (LifeGlobal ${ }^{\circledR}$ medium; IVFonline, Guilford, CT) covered with paraffin oil. After $5 \mathrm{~min}$, each sperm suspension was transferred to conditioned medium for preincubation. The control conditioned medium for sperm preincubation was HTF medium containing $1 \mathrm{mg} / \mathrm{mL}$ polyvinyl alcohol (PVA; Sigma, St. Louis, MO) and $1.0 \mathrm{mM}$ methyl-beta-cyclodextrin (MBCD; Sigma) [5]. A total of $20 \mu \mathrm{L}$ of the sperm suspension in HTF without BSA was transferred to $20 \mu \mathrm{L}$ of each conditioned medium containing twice the concentration of PVA, MBCD, and licorice extract and kept at $37^{\circ} \mathrm{C}$ in a humidified incubator under $5 \%$ $\mathrm{CO}_{2} / 95 \%$ air $(\sim 10,000 / \mu \mathrm{L}$ of the motile sperm concentration). After $50 \mathrm{~min}, 2-4 \mu \mathrm{L}$ of sperm from each conditioned medium was used for insemination (final motile sperm concentration $=150 / \mu \mathrm{L})$. Motile sperm swimming at the periphery of each drop were used for insemination as described previously [5].

\section{IVF}

Female mice were superovulated by an intraperitoneal injection of $5 \mathrm{IU}$ of pregnant mare serum gonadotropin (Asuka Inc., Tokyo, Japan), followed 46-48 h later by $5 \mathrm{IU}$ of human chorionic gonadotropin (Asuka Inc.), and then euthanized 14-16 $\mathrm{h}$ thereafter. The mice were sacrificed by cervical dislocation just before the start of the experiment. Ovaries with oviducts were transferred to a $30 \mathrm{~mm}$ dish filled with paraffin oil (Nacalai Tesque, Kyoto, Japan). Cumulus-oocyte complexes were obtained from the ampullae of uterine tubes and transferred to dishes, each containing a $200 \mu \mathrm{L}$ drop of HTF medium covered with paraffin oil, under a stereomicroscope. Two to four cumulus-oocyte masses were transferred to each $200 \mu \mathrm{L}$ drop of HTF medium covered with paraffin oil for insemination. A sperm suspension cultured in conditioned medium was transferred to the insemination drop. At $24 \mathrm{~h}$ after insemination, the fertilization rate was determined as the proportion of 2-cell-stage embryos among all of the oocytes. After incubation in KSOM medium for an additional $48 \mathrm{~h}$, the blastocysts were implanted into the uteri of pseudopregnant females.

\section{Observation of the Acrosome Reaction}

Mature sperm from the caudal epididymis were incubated in HTF medium supplemented with BSA or HTF medium containing PVA plus $\mathrm{MBCD}$ at $37^{\circ} \mathrm{C}$ in a humidified incubator under $5 \% \mathrm{CO}_{2} / 95 \%$ air. Sperm were removed chronologically from the medium and were spotted onto microscope slides. The sperm samples were fixed through incubation with $70 \%$ methanol at $-20^{\circ} \mathrm{C}$ for $5 \mathrm{~min}$. The slides were then treated with $20 \mu \mathrm{g} / \mathrm{mL}$ FITC-conjugated peanut agglutinin (FITC-PNA) (Sigma) and examined under a fluorescence microscope. The status of the acrosome was evaluated by staining with FITC-PNA, which binds to the outer acrosomal membrane and, therefore, does not stain acrosome-reacted sperm [6].

\section{Statistical Analysis}

Differences between the experimental and control conditions were tested by a one-way analysis of variance and Fisher's protected least significant difference tests. All percentages were arc-sin-transformed before analysis. Significant differences $(P<0.05)$ are discussed here.

\section{RESULTS}

\section{Fertilization Rate}

The fertilizing ability of the sperm from wild-type C57BL/6 mice was high when using standard medium (e.g., HTF) supplemented with BSA of a suitable type for IVF or with PVA plus MBCD. It became evident that when the sperm of aged BALB/cA mice ( $>48$ weeks of age) were used [7], IVF was difficult and the fertilizing ability of the sperm varied between mice. We tested whether the fertilization rate was increased by the addition of licorice extract $(0.12 \mathrm{mg} / \mathrm{mL})$. The fertilization rate for HTF medium containing PVA plus MBCD and licorice extract is shown in Fig. (1a and b). Five separate experiments were conducted using five different $\mathrm{BALB} / \mathrm{cA}$ mice. In case $\mathrm{A}$, the fertilization rate was $0.0 \%$ when licorice-free medium containing $0.25 \%$ DMSO was used. When medium containing licorice extract was used, the fertilization rate improved to $15.6 \%$. In case $\mathrm{B}$, the increase was from 8.0 to $31.3 \%$; in case $\mathrm{C}$, the increase was from 43.5 to $84.0 \%$; in case D, it improved from 18.5 to $38.1 \%$; and in case E, the rate improved from 4.2 to $47.1 \%$. These results show that the fertilization rate increased in samples containing HTF medium and licorice extract. The optimal concentration of licorice extract in the HTF medium was $0.3 \mathrm{mg} / \mathrm{mL}$ (Fig. 2). The fertilization rate for HTF medium containing PVA plus MBCD and licorice extract at a concentration of $0,0.05,0.1$, $0.2,0.3$, or $0.5 \mathrm{mg} / \mathrm{mL}$ was $5.3 \pm 9.2,8.3 \pm 14.4,12 \pm 6.3$, $55 \pm 16.8,64 \pm 6.4$, and $28 \pm 20.0 \%$, respectively. Glycyrrhizin is the main component of licorice. The fractions containing glycyrrhizin and other compounds were separated and their activity was investigated. It became clear that the activity was not based on glycyrrhizin, but on the fraction containing flavonoids and other components (Fig. 3). Furthermore, we examined the viability of embryos treated with licorice extract. Females became pregnant and delivered live young after transplantation (Fig. 4).

\section{The Acrosome Reaction and Motility}

The acrosome reaction is an important event for fertilization. Therefore, we examined the effect of licorice on the acrosome reaction. We applied standard culture conditions for mouse IVF using sperm from C57Black6 and 


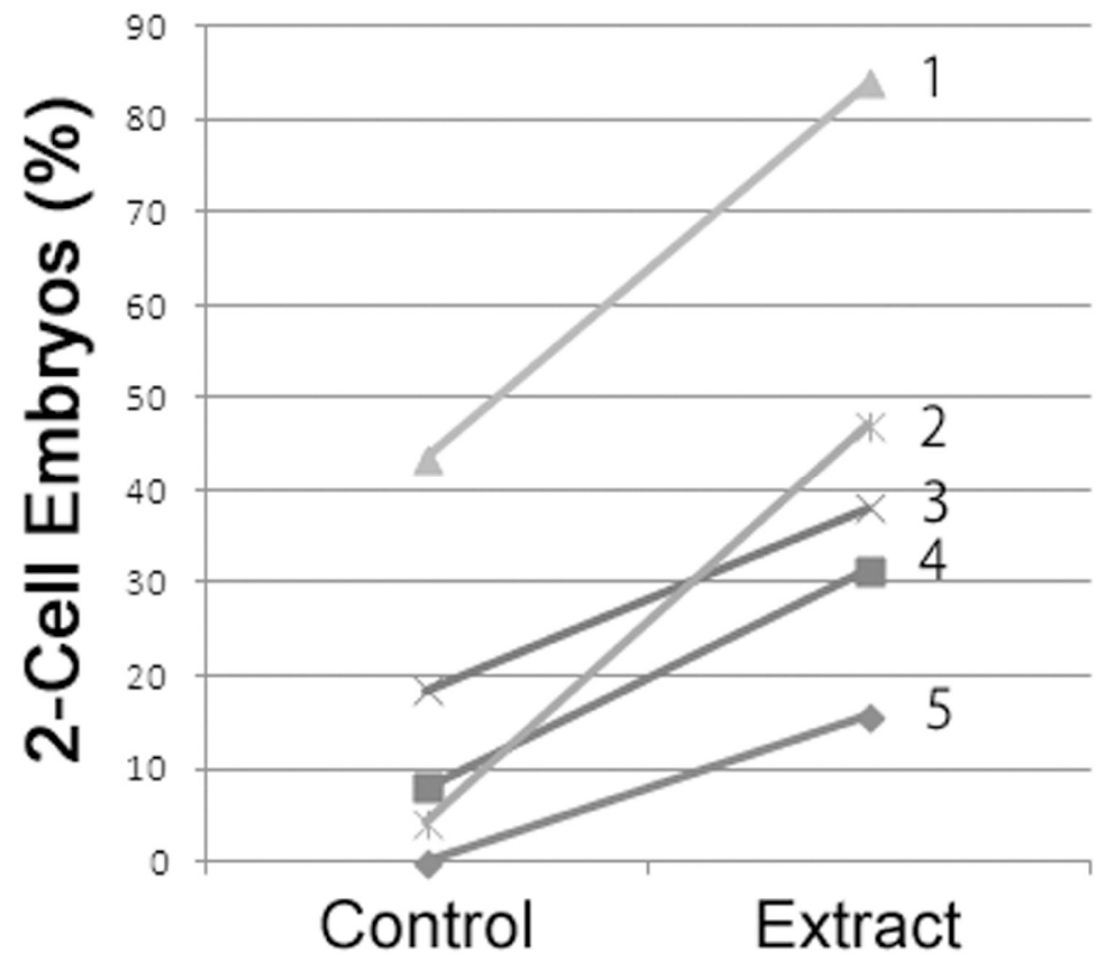

A

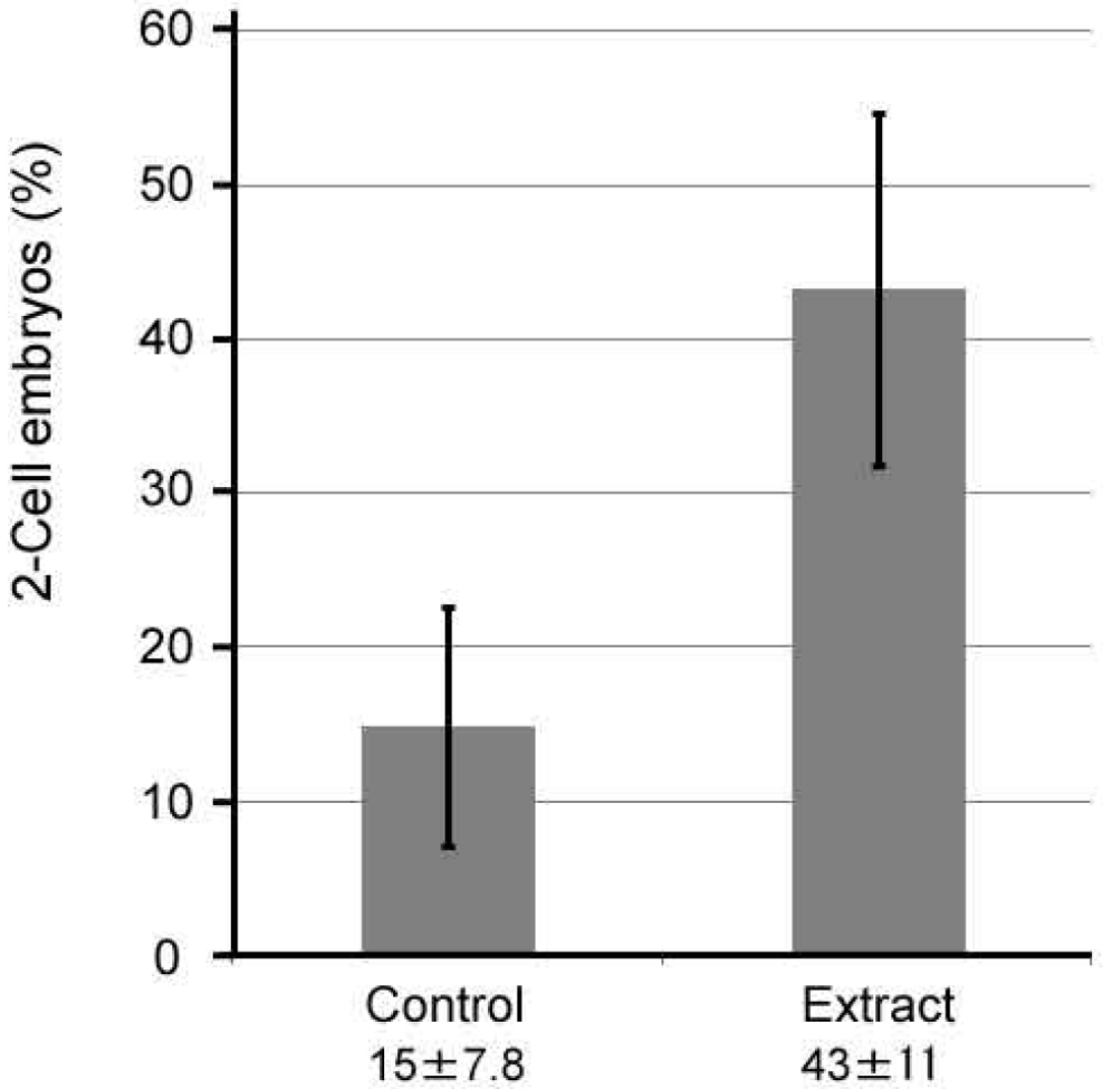

B

Fig. (1). Effect of licorice extract on the IVF rate. Sperm from BALB/cA mice were preincubated in conditioned medium with or without licorice extract $(0.12 \mathrm{mg} / \mathrm{mL})$. The fertilization rate varied among the male BALB/cA mice. In five experiments (1-5), more embryos were obtained with preincubation medium containing licorice extract (A) than with preincubation medium lacking licorice extract. The extract had a significant effect on IVF $(\mathbf{B})(P<0.05)$. 


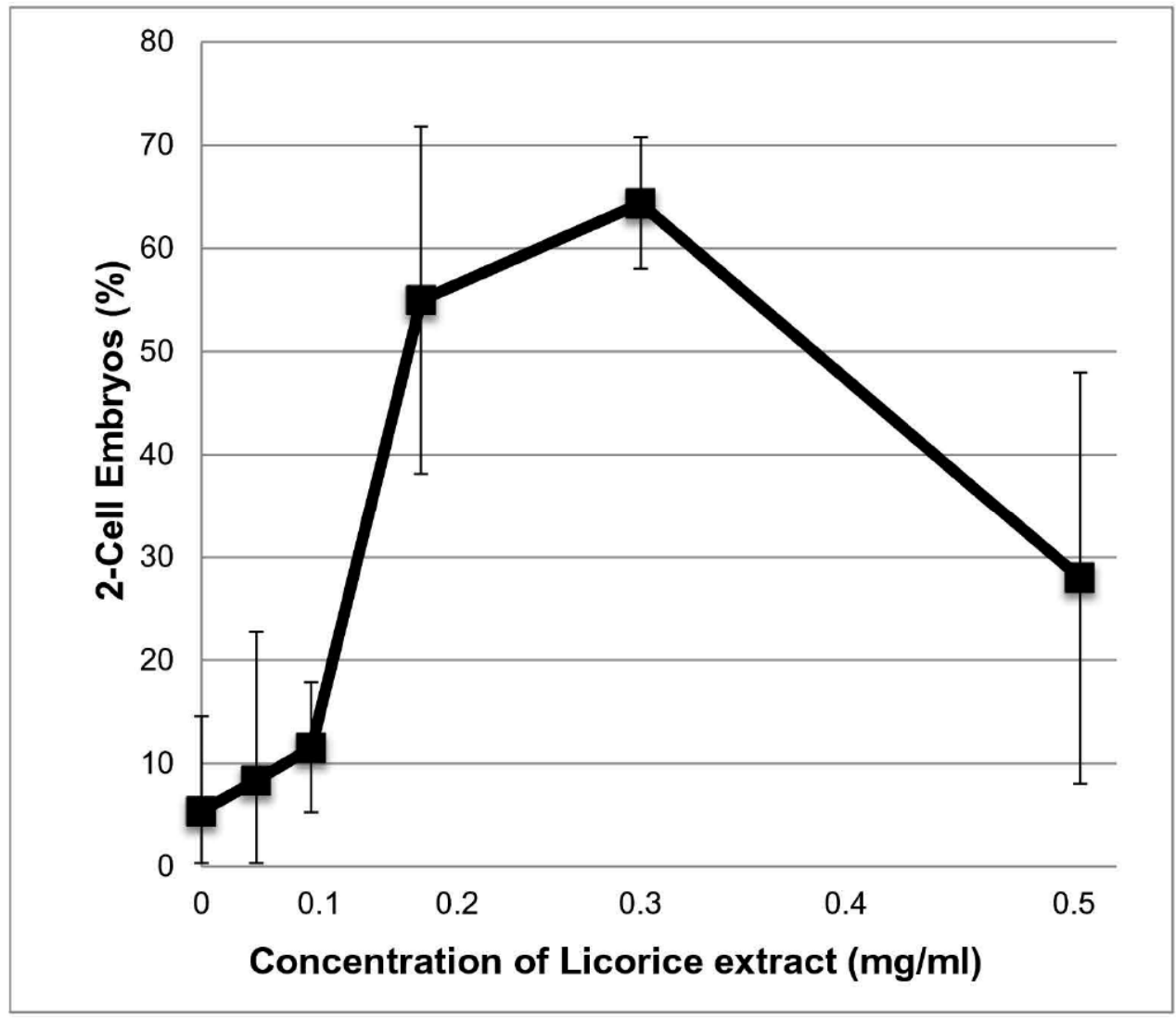

Fig. (2). Optimal concentration of licorice extract for IVF. The data are given as the means $\pm 95 \%$ confidence intervals of three experiments.

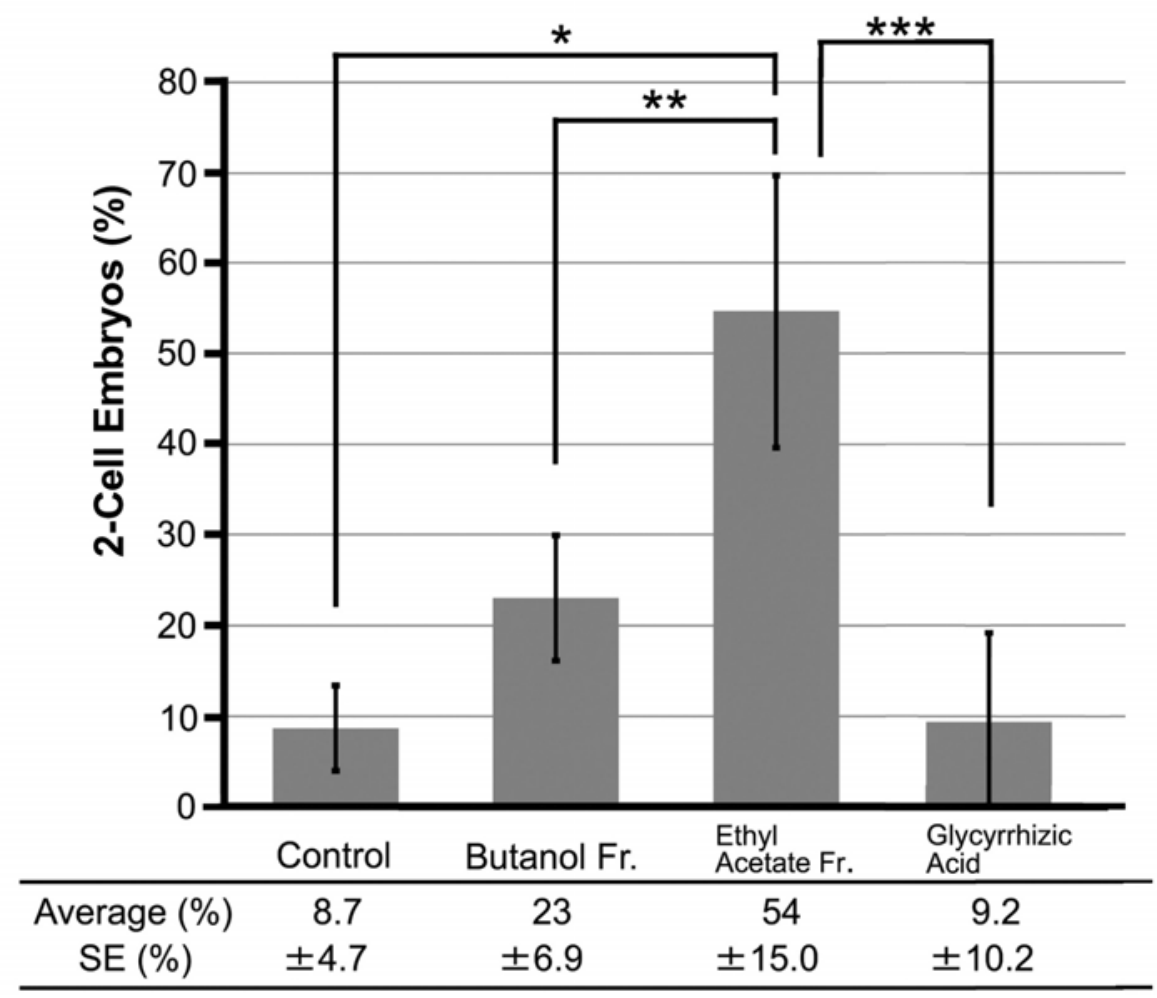

Fig. (3). Active constituent of the licorice extract for IVF. Butanol $(1.6 \mathrm{mg} / \mathrm{mL})$ and ethylacetate $(1 \mathrm{mg} / \mathrm{mL})$ extracts of licorice and glycyrrhizic acid (Sigma; product no. 50531) $(1.6 \mathrm{mg} / \mathrm{mL})$ were added to the conditioned medium. Activity was observed in the fraction (ethylacetate) containing flavonoids. *,*, and ***. The data are given as the means $\pm 95 \%$ confidence intervals of three experiments. 

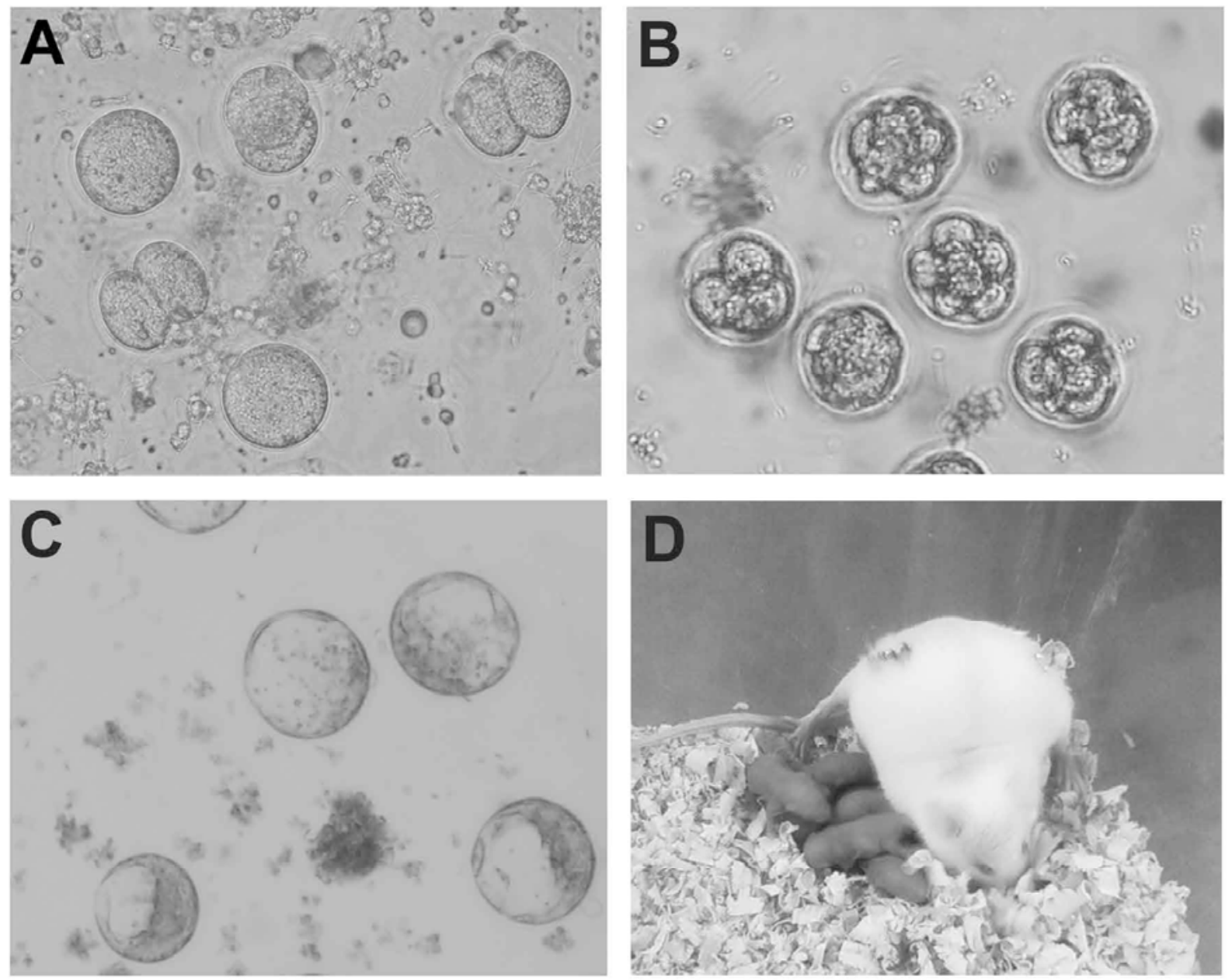

Fig. (4). Production of progeny from oocytes incubated with licorice extract. Two-cell-stage embryos (A), morulae (B), and blastocysts (C) after insemination in the presence of licorice extract. (D) Newborn mice delivered from the embryos shown in (C) after transfer to pseudopregnant females.

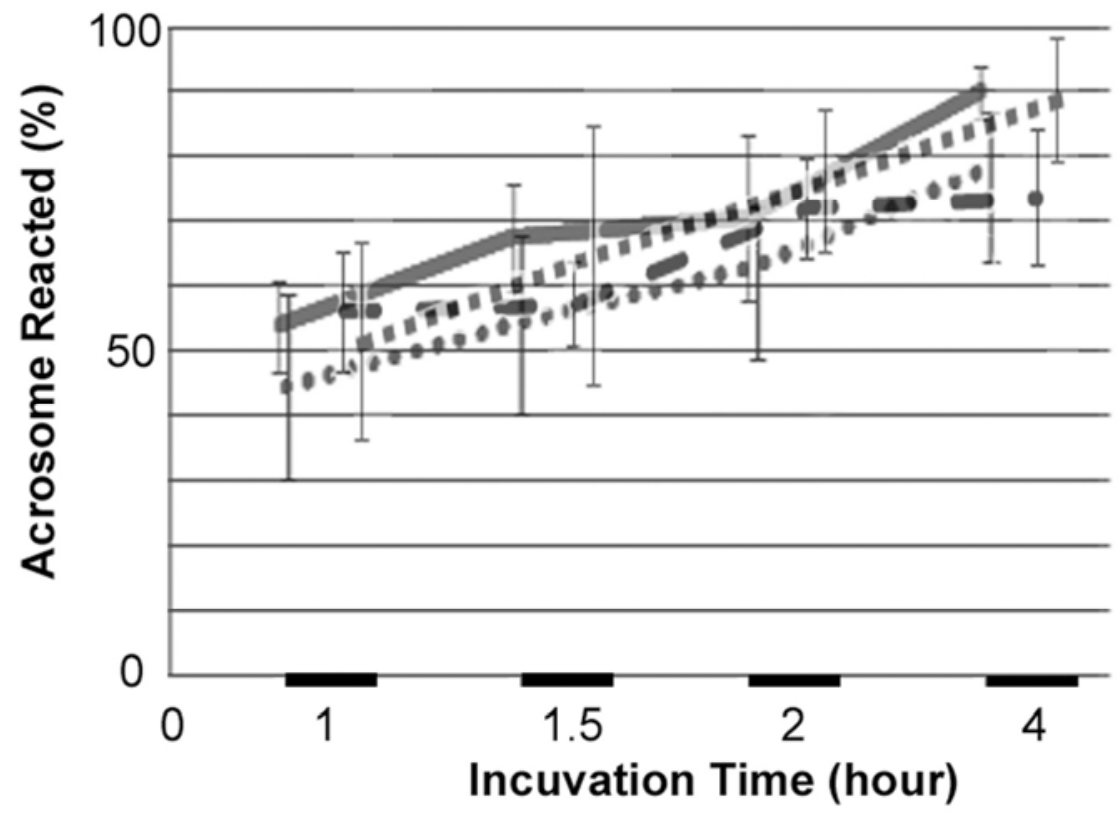

C57/B6 -

C57/B6 1000

with licorice

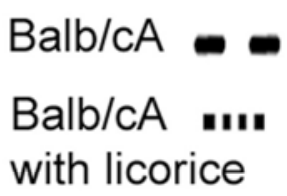

Fig. (5). The acrosome reaction in sperm incubated with or without licorice extract. Acrosome-reacted sperm were observed as described in the Materials and Methods. The data are given as the means $\pm 95 \%$ confidence intervals of three experiments $(* P<0.05)$. 
Table 1. Time-course analysis of the acrosome reaction in the presence of licorice extract.

\begin{tabular}{|c|c|c|c|c|}
\hline & \multicolumn{4}{|c|}{ Time After Preincubation (h) } \\
\hline Mouse Strains and Media & $\mathbf{1}$ & $\mathbf{1 . 5}$ & $\mathbf{2}$ & $\mathbf{4}$ \\
\hline \hline C57Black6 without licorice extract & $54 \pm 6.9$ & $68 \pm 13.8$ & $70 \pm 12.9$ & $63 \pm 9.0$ \\
\hline C57Black6 with licorice extract & $44 \pm 14.5$ & $54 \pm 13.1$ & $76 \pm 11.2$ & $79 \pm 8.3$ \\
\hline BALB/cA without licorice extract & $51 \pm 15.0$ & $64 \pm 20.1$ & $72 \pm 7.5$ & $89 \pm 9.5$ \\
\hline BALB/cA with licorice extract & $56 \pm 9.2$ & $57 \pm 6.4$ & & $74 \pm 10.5$ \\
\hline
\end{tabular}

The values for acrosome-reacted sperm are given as percentages.

The data are presented as means \pm SEM.

$\mathrm{BALB} / \mathrm{cA}$ mice. The reaction rate was not affected by licorice extract in HTF medium with or without PVA and MBCD (Fig. 5 and Table 1). These results indicate that the extract did not induce the acrosome reaction under standard IVF conditions.

\section{DISCUSSION}

Of the nearly 500 components contained in licorice root, the main active ingredients are glycyrrhizin and several flavonoids. To confirm this, we measured the content of glycyrrhizin in licorice root by Eastern blotting [8, 9] using an anti-glycyrrhizin monoclonal antibody, which resulted in a clear appearance. Therefore, licorice containing higher concentrations of glycyrrhizin is needed for phytotherapy. Although the ingestion of too much glycyrrhizin may cause steroid hormone-like activity and an allergic reaction to licorice, licorice is very safe and is eaten all around the world as a sweetener. Because licorice, which contains glycyrrhizin, has many pharmacological activities, the question of whether licorice can increase pregnancy rates has been investigated; however, no such increase has been reported [2]. In the present study, we found that licorice extract improved the fertilizing ability of BALB/cA mouse sperm in vitro and that the fertilized eggs developed normally. It was reported that licorice extract increased cyclophosphamide teratogenicity and upregulated the mRNA expression of cytochrome P-450 2B in rats [10]. These results indicate that licorice root contains multiple components with biological activity. These components may not affect gene expression because transcription rates in spermatozoa are low [11]. The addition of glycyrrhizin alone to the medium did not improve the fertilization ability of the sperm. Our results indicate that substances in licorice other than glycyrrhizin, or other substances plus glycyrrhizin, can improve IVF rates without damaging fertilized eggs. Future studies should be conducted to purify and test other active components of licorice root.

The licorice extracts maintained sperm mobility without inducing the acrosome reaction among sperm in the medium. The mechanisms underlying the maturation of ejaculated sperm remain unclear [2]. Hence, it is expected that the signals required for the acrosome reaction and for sperm motility are different, even if they are associated with each other.
The production of genetically modified mice has been indispensable for the study of gene function in vivo, and many genetically engineered strains have been obtained. Because modified lineages of mice are important for research, it is necessary to develop an easy way to preserve them. Moreover, although natural mating is possible, some lines show very poor IVF efficiency [4]; thus, it would be useful to improve IVF culture media. In the present study, the effects of licorice were investigated using sperm from $\mathrm{BALB} / \mathrm{cA}$ mice, which have a low fertilization rate, and improvement in the fertilization rate was detected. It would be interesting to know whether this extract can be used to improve fertilization in other animals, including humans.

\section{CONFLICT OF INTEREST}

The authors confirm that this article content has no conflict of interest.

\section{ACKNOWLEDGEMENTS}

Declared none.

\section{REFERENCES}

[1] Uto T, Tung NH, Morinaga O, Shoyama Y. Preparation of knockout extract by immune affinity column and its application. Antibodies 2011; 1: 294-307.

[2] Shin S, Jang JY, Choi BI, et al. Licorice extract does not impair the male reproductive function of rats. Exp Anim 2008; 57: 11-7.

[3] Yanagimachi R. Germ cell research: a personal perspective. Biol Reprod 2009; 80: 204-18.

[4] Yamashita M, Honda A, Ogura A, et al. Reduced fertility of mouse epididymal sperm lacking Prss21/Tesp5 is rescued by sperm exposure to uterine microenvironment. Genes Cells 2008; 13: 1001-13.

[5] Takeo T, Hoshii T, Kondo Y, et al. Methyl-beta-cyclodextrin improves fertilizing ability of C57BL/6 mouse sperm after freezing and thawing by facilitating cholesterol efflux from the cells. Biol Reprod 2008; 78: 546-51.

[6] Kitamura K, Tanaka H, Nishimune Y. Haprin, a novel haploid germ cell-specific RING finger protein involved in the acrosome reaction. J Biol Chem 2003; 278: 44417-23.

[7] Vasudevan K, Sztein JM. Treatment of sperm with extracellular adenosine 5'-triphosphate improves the in vitro fertility rate of inbred and genetically modified mice with low fertility. Theriogenology 2011; 76: 729-36.

[8] Shan S, Tanaka H, Shoyama Y. Western blotting method for the immunostaining detection of glucuronides of glycyrrhetic acid using anti-glycyrrhizin monoclonal antibody. Biol Pharm Bull 1999; 22: 221-3. 
[9] Shan S, Tanaka H, Shoyama Y. Enzyme-linked immunosorbent assay for glycyrrhizin using anti-glycyrrhizin monoclonal antibody and an eastern blotting technique for glcucuronides of glycyrrhetic acid. Anal Chem 2001; 73: 5784-90.

[10] Park D, Yang YH, Choi EK, et al. Licorice extract increases cyclophosphamide teratogenicity by upregulating the expression of cytochrome P-450 2B mRNA. Birth Defects Res B Dev Reprod Toxicol 2011; 92: 553-9.

[11] Tanaka H, Baba T. Gene expression in spermiogenesis. Cell Mol Life Sci 2005; 62: 344-54.

Received: January 10, 2014

Revised: April 21, 2014

Accepted: May 09, 2014

(C) Tung et al.; Licensee Bentham Open.

This is an open access article licensed under the terms of the Creative Commons Attribution Non-Commercial License (http://creativecommons.org/licenses/by-nc/3.0/) which permits unrestricted, non-commercial use, distribution and reproduction in any medium, provided the work is properly cited. 